\title{
Check-list of the Chironomidae (Diptera) of the river Meuse and two of its tributaries
}

\author{
M. Evrard 1
}

Keywords : Diptera, Chironomidae, Belgium, France, faunistic, river, Meuse.

A study of the Chironomidae (Diptera) of the river Meuse and two of its tributaries (Sambre and Samson) during the period 1991 to 1992 resulted in a list of one hundred and twenty species. Thirty one of these $\left(^{*}\right)$ are new records to the Belgian fauna. A taxonomic list of the species with locations is given.

Inventaire des Chironomidés (Diptera) de la Meuse et de deux de ses affluents.

Mots Clés : Diptera, Chironomidés, Belgique, France, faunistique, rivière, Meuse.

Des récoltes d'exuvies nymphale de Chironomidés effectuées en 1991 et 1992 dans la Meuse et deux de ses affluents (la Sambre et le Samson) ont permis de dénombrer 120 espèces de Chironomidés. Il s'agit, pour 31 espèces $\left({ }^{*}\right)$, de premières citations pour la faune belge. L'inventaire taxonomique des espèces et la localisation des sites d'échantillonnage sont fournis.

\section{Introduction}

The Chironomidae is a family of non-biting midges of the order Diptera. So far studies on the Belgian Chironomidae yielded about three hundred species (Goddeeris \& Behen 1991). However, most of the earliest studies were concentrated on ponds and temporary pools located mostly in Flanders. The present study was to understand the species diversity of chironomids in the river Meuse (and two of its tributaries) to be used as water quality indicators of the river.

During several centuries, the river Meuse has been used for different human activities. Consequently habitats and water quality have been heavily modified. In Belgium, the main impacts on the biocenoses are due to the equipment of the river for navigation, but many other kinds of pollution (thermal, chemical) also have some effects on these biocenoses (Meurisse-Genin et al. 1987).

\footnotetext{
1. Unité d'Ecologie des Eaux Douces (UNECED), Facultés Universitaires Notre-Dame de la Paix, rue de Bruxelles 61, B-5000 Namus, Belgium.
}

Previous work by Frantzen (1992) has shown that collections of Chironomidae pupal exuviae may be related to the water quality of the river Meuse. The predominant taxa-collected in the belgian Meuse show the difference of water quality between Hastière and the Liège area. Hastière, the most upstream belgian site, is the least polluted with rheophilic species as Rheotanytarsus rhenanus, $R$. photophilus, Tanytarsus eminulus and $T$. ejuncidus. In the Liège area, the dominant species (Procladius spp., Dicrotendipes nervosus, Glyptotendipes pallens, Parachironomus arcuatus) are indicative for slowing and eutrophic waters.

\section{Materiel and methods}

The Meuse rises near the Plateau of Langres in France. The it flows through France, Belgium and the Netherlands, where it enters the North Sea by forming with the Rhine and Schelt a complex system of channels, nowadays entirely dammed for flood control (delta plan). Its total catchment area extends through 5 countries : France, Belgium ( $42 \%$ of the Meuse watershed), the Netherlands, Germany and Luxembourg (Fig. 1). 

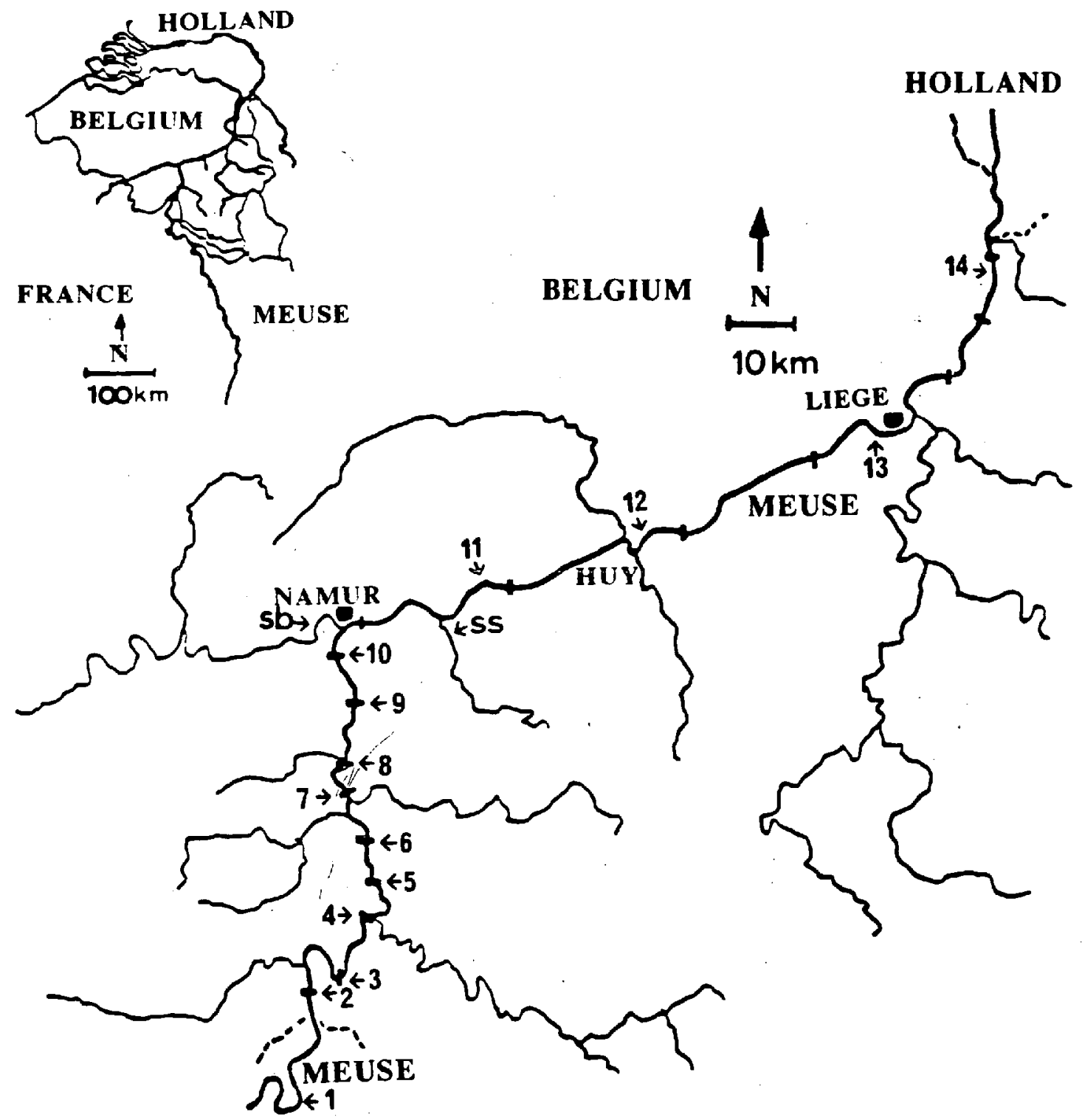

\section{FRANCE}

Fig. 1. Map showing the sampling sites (1 to 14) in the river Meuse and two of its tributaries (sb = Sambre; ss = Samson). The site 1 only is situated in France.

Fig. 1. Emplacement des stations (1 à 14) de la rivière Meuse et de deux affluents ( $s b=$ Sambre ; $s s=$ Samson). Seule la station 1 est située en France. 
Table 1. List of the Diptera Chironomidae in the river Meuse and two of its tributaries.

* = new record for Belgium ;

1 to $\mathbf{1 4}=$ stations in the river Meuse (Fig. 1 ); $\mathbf{s b}=$ Sambre ; ss = Samson.

Tableau 1. Liste des Chironomidés de la rivière Meuse et deux de ses affluents.

* = nouvelle récolte pour la Belgique ;

1 à 14 = stations de la rivière Meuse (Fig. 1); sb = Sambre ; ss = Samson.

\section{Chironomidae}

\section{Tanypodinae}

Ablabesmyiamonilis (Linnaeus)

Apsectrotanypus trifascipennis (Zetterstedt)

Conchapelopia melanops Wiedemann (in Meigen)

Conchapelopia pallidula (Meigen)

Guttipelopia guttipennis (van der Wulp)

Macropelopia nebulosa (Meigen)

Nilotanypus dubius (Meigen)

Procladius (Holotanypus) choreus (Meigen)

Procladius (Holotanypus) sagittalis (Kieffer)

Bheopelopia ornata (Meigen)

Thienemannimyia carnea (Fabricius)

* Thienemannimyia vitellina (Kieffer)

Zavrelimyia melanura (Meigen)

\section{Stations}

1 to $10, \mathrm{ss}$

2 to $12, \mathrm{ss}$

$2,6,10$, ss

2 to 10

2

12 ,ss

$1,6,9,10$

1 to $14, \mathrm{sb}, \mathrm{ss}$

2 to $14, \mathrm{sb}$

1 to 10

$1,9,10$, ss

2 to 11

SS

SS

2

1 to $12, \mathrm{ss}$

2 to $13, \mathrm{sb}, \mathrm{ss}$

\section{Orthocladiinae}

* Brillia flavifrons Johannsen

$10,11,12$, sb,ss

Brillia modesta (Meigen)

1 to $12, \mathrm{sb}, \mathrm{ss}$

* Bryophaenocladius furcatus (Kieffer)

1 to $12, \mathrm{sb}$

Cardiocladius fuscus Kieffer

1 to $11, \mathrm{sb}$

Chaetocladius melaleucus (Meigen)

2

Chaetocladius perennis (Meigen)

$9,12,13$

Cricotopus (Cricotopus) albiforceps (Kieffer) 
Table 1. (continuation).

Tableau 1. (suite).

Cricotopus (Cricotopus) annulator Goetghebuer

Cricotopus (Cricotopus) bicinctus (Meigen)

Cricotopus (Cricotopus) similis Goetghebuer

Cricotopus (Cricotopus) tremulus (Linnaeus)

Cricotopus (Cricotopus) triannulatus (Macquart)

Cricotopus (Cricotopus) trifascia Edwards

Cricotopus (Cricotopus) vierriensis Goetghebuer

Cricotopus (Isocladius) intersectus (Staeger)

Cricotopus (Isocladius) sylvestris (Fabricius)

Diplocladius cultriger Kieffer

* Eukiefferiella brevicalcar (Kieffer)

Eukiefferiella claripennis (Lundbeck)

* Eukiefferiella clypeata (Kieffer)

* Eukiefferiella coerulescens (Kieffer)

Eukiefferiella devonica (Edwards)

Heterotrissocladius marcidus (Walker)

* Limnophyes pumilio (Holmgren)

Nanocladius bicolor (Zetterstedt)

* Nanocladius distinctus (Malloch)

* Nanocladius rectinervis (Kieffer)

Orthocladius (Eudactylocladius) fuscimanus (Kieffer)

* Orthocladius (Euorthocladius) ashei Soponis

* Orthocladius (Euorthocladius) rivulorum Kieffer Orthocladius (Orthocladius) glabripennis (Goetghebuer)

* Orthocladius (Orthocladius) oblidens (Walker)

* Orthocladius (Orthocladius) obumbratus Johannsen

* Orthocladius (Orthocladius) pedestris Kieffer Orthocladius (Orthocladius) rubicundus (Meigen)

* Orthocladius (Orthocladius) wetterensis Brundin Paracladius conversus (Walker) Paracricotopus niger (Kieffer)

* Parakiefferiella bathophila (Kieffer)

* Parakiefferiella Pel Langton 1991

* Parametriocnemus stylatus (Kieffer) Paratrichocladius rufiventris (Meigen)
1 to 12

1 to $14, \mathrm{sb}, \mathrm{ss}$

$1,2,9$, ss

1 to 12 ,ss

1 to 12 ,sb,ss

1 to 12 ,ss

$1,5,9$

1 to $14, \mathrm{sb}$

1 to $14, \mathrm{sb}$

11

ss

1 to 14, sb,ss

1 to 12 ,ss

SS

ss

2 to 12 ,ss

1 to $12, \mathrm{sb}$

1 to $14, \mathrm{sb}$,ss

1 to $13, \mathrm{sb}, \mathrm{ss}$

1 to $14, s b$,ss

1 to $13, \mathrm{sb}$

1 to $12, \mathrm{ss}$

$2,6,12$,ss

1 to 12 ,ss

1 to 13, sb,ss

1 to 14 ,ss

$2,6,11$

1 to $14, \mathrm{sb}$,ss

ss

$5,9,10,11,5 s$

$3,8,9,10,12, \mathrm{ss}$

12 ,ss

1 ,ss

1 to $12, \mathrm{sb}, \mathrm{ss}$

1 to $14, s b, s s$ 
Table 1. (continuation).

Tableau 1. (suite).

$\begin{array}{ll}\text { Paratendipes albimanus (Meigen) } & 1 \text { to } 12 \\ \text { Phaenopsectra flavipes (Meigen) } & 2 \text { to } 14 \\ \text { Polypedilum (Pentapedilum) sordens (van der Wulp) } & \text { ss } \\ \text { Polypedilum (Pentapedilum) uncinatum (Goetghebuer) } & 7 \\ \text { Polypedilum (Polypedilum) albicorne (Meigen) } & 6, s s \\ \text { Polypedilum (Polypedilum) convictum (Walker) } & 1 \text { to } 14, \mathrm{ss} \\ \text { Polypedilum -(Polypedilum) cultellatum Goetghebuer } & 1 \text { to } 14, \mathrm{sb}, \mathrm{ss} \\ \text { Polypedilum (Polypedilum) laetum (Meigen) } & 2 \text { to } 14, \mathrm{ss} \\ \text { Polypedilum_(Polypedilum) nubeculosum (Meigen) } & 1 \text { to } 14 \\ \text { Polypedilum (Tripodura) pullum (Zetterstedt) } & 1 \text { to } 10, \mathrm{ss} \\ \text { Polypedilum_(Tripodura) scalaenum (Schrank) } & 1 \text { to } 14 \\ \text { Xenochironomus xenolabis (Kieffer) } & 1,2,3,6,7,12\end{array}$

\section{Chironominae}

Tanytarsini

\author{
Cladotanytarsus mancus (Walker) \\ Micropsectra atrofasciata (Kieffer) \\ Micropsectra notescens (Walker) \\ * Paratanytarsus dissimilis Johannsen \\ Rheotanytarsus curtistylus (Goetghebuer) \\ * Rheotanytarsus pentapoda Kieffer \\ Rheotanytarsus photophilus (Goetghebuer) \\ Rheotanytarsus rhenanus Klink \\ * Stempellina almi Brundin \\ * Tanytarsus brundini Lindeberg \\ * Tanytarsus curticomis Kieffer \\ Tanytarsus ejuncidus (Walker) \\ Tanytarsus eminulus (Walker) \\ * Tanytarsus fimbriatus Reiss \& Fittkau \\ Tanytarsus heusdensis Goetghebuer \\ * Tanytarsus palletaris Verneaux \\ Tanytarsus pallidicormis (Walker)
}

\section{Stations}

1 to 10

2 to $14, s b, s s$

12 ,ss

1 to 12 ,sb,ss

2

1 to $10, \mathrm{sb}$

1 to 10

1 to 12 ,sb,ss

8

1 to 13 ,ss

2,11

1 to 12 ,ss

1 to $14, \mathrm{ss}$

5

1 to $14, \mathrm{sb}$

ss

$4,6,7,8$ 
Table 1. (continuation).

Tableau 1. (suite).

$\begin{array}{ll}\text { Psectrocladius (Psectrocladius) sordidellus (Zetterstedt) } & 1,2 \\ \text { Rheocricotopus (Psilocricotopus) chalybeatus (Edwards) } & 1 \text { to } 12, \mathrm{sb}, \mathrm{ss} \\ \text { Rheocricotopus (Rheocricotopus) fuscipes (Kieffer) } & 1 \text { to } 14, \mathrm{sb}, \mathrm{ss} \\ \text { Rheorthocladius sp. A Thienemann } & 6,9, \mathrm{ss} \\ \text { Smittia sp. } & 2,5,9, \mathrm{ss} \\ \text { Synorthocladius semivirens (Kieffer) } & 1 \text { to } 14, \mathrm{sb} \\ \text { Thienemanniella clavicomis (Kieffer) } & 1,3, \mathrm{ss} \\ \text { Tvetenia calvescens (Edwards) } & 1 \text { to } 12, \mathrm{ss} \\ \text { Tvetenia discoloripes (Goetghebuer) } & 1 \text { to } 11 \\ \text { Tvetenia verralli (Edwards) } & 1 \text { to } 12, \mathrm{sb}, \mathrm{ss}\end{array}$

\section{Chironominae}

\section{Chironomini}

\section{Stations}

* Chironomus (Chironomus) anthracinus Zetterstedt

$1,6,9,10,11,12$

Chironomus (Chironomus) aprilinus Meigen

$12,13,14$, sb,ss

Chironomus (Chironomus) plumosus (Linnaeus)

1 to 14

Chironomus (Chironomus) riparius Meigen

$13, \mathrm{sb}$

Cladopelma virescens (Meigen)

1 to 10

Cryptochironomus rostratus Kieffer

2 to 10

Cryptochironomus supplicans (Meigen)

1 to 12

Cryptotendipes pseudotener (Goetghebuer)

12

Dicrotendipes nervosus (Staeger)

1 to $14, \mathrm{sb}$

Endochironomus albipennis (Meigen)

1

Glyptotendipes pallens (Meigen)

1 to $14, \mathrm{sb}$

Glyptotendipes paripes Edwards

1 to $14, \mathrm{sb}$

* Glyptotendipes signatus Kieffer

3

Hamischia curtilamellata (Malloch)

1 to 12

Kiefferulus tendipedifomis (Goetghebuer) 3

Microchironomus tener (Kieffer)

1 to 12

Microtendipes chloris (Meigen)

1 to 12 ,ss

Microtendipes pedellus (De Geer)

$12, \mathrm{sb}$

Nilothauma brayi (Goetghebuer)

$3,7,10$

Parachironomus arcuatus (Goetghebuer)

1 to $14, \mathrm{sb}, \mathrm{ss}$

Parachironomus frequens (Johannsen)

1 to 14

Parachironemus vitiotus (Goetghebuer) 
A set of 8 samples were taken between the French border and Namur (In fact at each dam) with 10 to $15 \mathrm{~km}$ intervals. One sample was taken in France and four samples downstream Namur. Two samples were also taken in two tributaries of the river Meuse : the Sambre (a polluted river) and the Samson (a clean stream). Monthly sampling of chironomid exuviae was done and samples were fixed in $95 \%$ alcohol and were sorted in the laboratory. All the sites on the river Meuse were visited during the same day. Pupal exuviae of chironomids were sorted in the samples and identified to species level with the Wilson \& Mc Gill 1982 and Langton 1991 keys. For identification exuviae were mounted in Euparal in permanent slides.

\section{Results}

Monthly samples collected from the river Meuse and its tributaries for a two years period yielded a total of 120 species of Chironomidae ; their list is given in the Table 1 .

\section{Conclusion}

Of the one hundred and twenty species recorded during this survey, thirty one are new to the fauna of Belgium (see Goddeeris \& Behen (1991), for details). All the new records are being prepared for a detailed taxonomic description.

\section{Acknowledgments}

I am thankful to Prof. J.C. Micha for helpful comments on the manuscript and for providing facilities and financial support.

\section{References}

Frantzen N. 1992. - Water quality changes of the river Meuse assessed by Chironomid pupal exuviae. Netherlands Journal of Aquatic Ecology, 26 (2-4) : 543-549.

Goddeeris B. \& Behen F. 1991. - Chironomidae. In « Catalogue of the Diptera of Belgium » by Grootaert P., De Bruyn L. \& De Meyer M. Documents de travail 70 de l'Institut Royal des Sciences Naturelles de Belgique, Bruxelles : 46-56.

Langton P.H. 1991. - A key to pupal exuviae of West Palaearctic Chironomidae P.H. Langton ed. Huntington, Cambridgeshire, England : 386 p.

Meurisse-Genin M., Reydamss-Detollenaere A., Stroot Ph. \& Michal J.C. 1987. - Les macroinvertébrés benthiques de la Meuse belge : bilan de cinq années de recherche, (1980 à 1985). Arch. Hydrobiol., $109: 67-88$.

Wilson R.S. \& Mc Gill J.D. 1982. - A practical key to the genera of pupal exuviae of the British Chironomidae (Diptera, Insecta). Univ. Bristol Publ. U.K. : 62 p. 